\title{
Transatlantica
}

Revue d'études américaines. American Studies Journal

1 | 2017

Morphing Bodies: Strategies of Embodiment in Contemporary US Cultural Practices

\section{Jackson Pollock, Untitled (c.1938-1941) - The Shape of Things to Come}

\section{Catherine Marcangeli}

\section{(2) OpenEdition}

\section{Journals}

Édition électronique

URL : https://journals.openedition.org/transatlantica/8460

DOI : $10.4000 /$ transatlantica. 8460

ISSN : 1765-2766

Éditeur

Association française d'Etudes Américaines (AFEA)

Référence électronique

Catherine Marcangeli, « Jackson Pollock, Untitled (c.1938-1941) - The Shape of Things to Come », Transatlantica [En ligne], 1 | 2017, mis en ligne le 03 décembre 2018, consulté le 22 mai 2021. URL : http://journals.openedition.org/transatlantica/8460; DOI : https://doi.org/10.4000/transatlantica. 8460

Ce document a été généré automatiquement le 22 mai 2021.

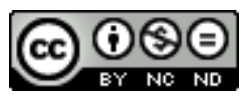

Transatlantica - Revue d'études américaines est mise à disposition selon les termes de la licence Creative Commons Attribution - Pas d'Utilisation Commerciale - Pas de Modification 4.0 International. 


\section{Jackson Pollock, Untitled (c. 1938-1941) - The Shape of Things to Come}

\section{Catherine Marcangeli}

Jackson Pollock (1912-1956) @ ARS, NY. Untitled, c.1938/41

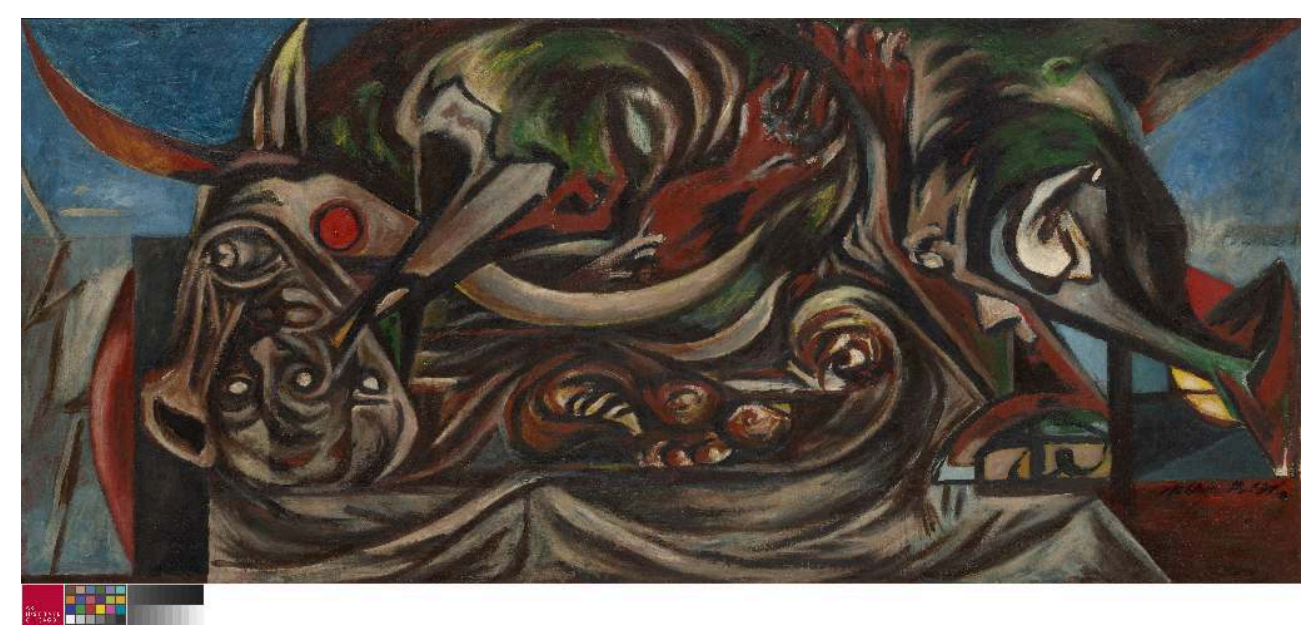

OIL ON LINEN. 56,5 X 127,6 cM (22 1/4 X 50 1/4 IN.), UNFRAMED. MAJOR ACQUISITIONS CENTENNIAL FUND; ESTATE OF FLORENE MAY SCHOENBORN; THROUGH PRIOR ACQUISITIONS OF MR. AND MRS. CARTER H. HARRISON, MARgUERITA S. RITMAN, MR. AND MRS. BRUCE BORLAND, AND MARY L. AND LEIgH B. BLOCK, 1998.522.

PHOTO CREDIT: THE ART INSTITUTE OF CHICAgO / ART RESOURCE, NY

1 L'exposition The Age of Anxiety, présentée au Musée de l'Orangerie fin 2016, couvrait la période allant de la Grande Dépression à la Deuxième Guerre Mondiale. Écrit lors de l'arrivée de W.H. Auden à New York en 1939, et publié en 1947, le poème qui lui a donné son titre est une méditation sur la condition humaine, sur la solitude, la perte et 
l'espoir. Cette anxiété est soulignée dès les premières lignes du communiqué de presse et du texte d'introduction de l'exposition : «Le 29 octobre 1929, la bourse de New York s'effondra, entraînant les Etats-Unis dans une terrible période d'insécurité économique et de troubles sociaux, la Grande Dépression. » Dans le même communiqué de presse, les termes "destabilisation ", " questionnements » et " temps difficiles " sont utilisés pour décrire les années trente, et l'exposition se donnait pour mission de montrer comment les artistes américains ont réagi face à cette situation.

Untitled (c.1938-1941, Chicago Art Institute) était le seul tableau de Jackson Pollock inclus dans The Age of Anxiety. Les fragmentations et déformations des corps représentés avaient certainement leur place dans une exposition qui explore les tourment sociaux, psychologiques et stylistiques de cette décennie. Néanmoins, pour le visiteur, l'accrochage de cette toile était aussi frappant que ses caractéristiques formelles : elle était en effet accrochée placée ? dans la dernière salle, à côté de la célèbre station essence d'Edward Hopper, Gas (1940, MoMA, New York). On peut imaginer que cette décision des conservateurs tenait en partie à la date d'exécution du tableau, 1938-1941, à cheval entre les années trente et les années quarante. La présence de ces deux toiles, côté à côte, seules face à un mur vide, semblait toutefois surprenante : pourquoi ce Pollock-là, plutôt qu'un autre ? Et pourquoi ce Pollock accroché là, en conclusion?

\section{The Age of Anxiety}

Untitled est une image fragmentée. La tête de taureau est comme disloquée, la tête humaine (en bas à gauche) est à l'envers, et dédoublée, les becs et les cornes s'enchevêtrent et se confondent.

4 Plusieurs mouvements circulaires animent les formes, créant des tourbillons interrompus par des lignes droites - dont la table et les angles droits qui débordent à gauche et à droite de la composition. L'espace est lui aussi fragmenté, et notre point de vue en est déstabilisé. De nombreuses diagonales semblent construire une perspective géométrique, mais elles brisent l'espace plus qu'elles ne le définissent. Le spectateur a du mal à appréhender l'organisation spatiale de la toile : l'avant-plan et l'arrière-plan se télescopent, la table penche vers l'avant comme dans certaines natures mortes de Cézanne, prête à basculer, croulant sous le poids des masses humaines et animales. Les couleurs contribuent à ce déséquilibre. Les couleurs primaires (vert, rouge) et secondaires (bleu) ne se complètent pas; elles sont impures, presque sales, en disharmonie. Des touches de rouge sont appliquées à intervalles réguliers pour ancrer la composition, mais elles perturbent la lecture de l'espace en créant une succession d'avant-plans arbitraires, participant à ce que Hans Hoffmann décrivait comme un mouvement de «push and pull» - on ne sait pas par exemple à quoi correspond le cercle rouge à côté de l'œil du bœuf, mais il se trouve propulsé vers l'avant.

Ces caractéristiques formelles ont souvent été interprétées comme la marque des tourments intérieurs de l'artiste, et comme leur symptôme et leur expression. En effet, de 1939 à 1940, Pollock consulte Joseph Henderson, analyste jungien qui l'encourage à dessiner. Les 83 dessins produits pendant cette période explorent une angoisse existentielle, exacerbée par le contexte politique international, les effets de la Guerre Civile espagnole, les débuts de la Seconde Guerre mondiale. Dans ce tableau sans titre 
aussi, les dislocations sont synonymes d'une violence et d'une souffrance dont la cause n'est pas clairement identifiée.

Untitled est traversé de références à d'autres œuvres et mouvements artistiques. Ce genre de relation, sur le mode du modèle, du dialogue ou du rejet, était examiné dans l'exposition, qui visait à montrer comment les artistes américains des années trente commencèrent à "élaborer un art propre à cette nation encore jeune" (texte d'introduction de la salle intitulée «L'histoire revisitée »).

7 Dans certaines toiles de Pollock comme Masqued Image (1938), les disharmonies chromatiques, les distorsions ou fragmentations des corps et les visages semblables à des masques suggèrent l'influence de Picasso. Untitled, même si sa taille est modeste, a par exemple de nombreux points communs avec Guernica (1937) : le format horizontal très allongé de la toile, le taureau et le cheval, les figures et les contorsions. Pollock connaissait bien l'œuvre de Picasso : en 1936, l'exposition Cubism and Abstract Art s'était tenue au MoMA ; en 1939, Pollock avait vu Guernica à la Galerie Valentine Dudensing après avoir fait sensation à Paris en 1937, le tableau était en tournée pour récolter des fonds contre le fascisme. Fin 1939-début 1940, l'exposition Picasso, 40 Years of His Art, au MoMA incluait Guernica et Les Demoiselles d'Avignon.

8 Des affinités avec le Surréalisme sont également perceptibles dans l'œuvre de Pollock. Si certains membres du groupe surréaliste ont séjourné à New York pendant la Seconde Guerre mondiale, certains d'entre eux avaient exposé dès le milieu des années trente dans des galeries new-yorkaises, dont la galerie Pierre Matisse (De Chirico en 1935, Miró tous les ans à partir de 1936) ou la galerie Julien Levy (Dalì en 1936). Quant à l'exposition Fantastic Art: Dada and Surrealism, elle avait eu lieu au MoMA en 1937. Untitled offre une vision cauchemardesque où animaux, humains et objets ont une identité instable et se métamorphosent. Dans The Moon Woman (1942), Pollock fait écho à Miró, à Picasso, ainsi qu'à Jung. Des hiéroglyphes et images totémiques sont également présents dès le début des années quarante dans les tableaux d'Adolf Gottlieb et de Mark Rothko, ou dans l'œuvre de Matta qui, à la même époque, pratique l'automatisme avec les peintres de New York. Pendant les années quarante, Pollock a exposé aux côtés de Surréalistes : en 1943 dans Natural, Insane, Surrealist Art à la galerie de Peggy Guggenheim, Art of This Century. Sidney Janis a également inclus Pollock dans l'exposition Abstract and Surrealist Art, en 1944, soulignant une parenté déjà explicite dans l'étiquette "Abstract Surrealism», parfois appliquée aux artistes Expressionnistes Abstraits.

Dans The Age of Anxiety, le panneau introductif intitulé «L'histoire revisitée » soulignait que de nombreux artistes américains se sont détournés des modèles européens pour découvrir leur spécificité et leur identité américaines. Même si à la fin des années trente Pollock s'éloigne du modèle régionaliste, Untitled peut être rapproché de certaines compositions allégoriques de Thomas Hart Benton, son mentor. Un mouvement tourbillonnant, l'intersection de courbes et de lignes droites, et l'interpénétration des plans caractérisent déjà des toiles comme People of Chilmark (Figure Composition), (1920, Hirshhorn Museum collection, Washington, D.C.). Quant à Invasion (1942, State Historical Society of Missouri, Columbia), c'est une œuvre plus tardive qui fait partie de la série Year of Peril, peinte à la suite de Pearl Harbour ; là aussi, les plans s'entrechoquent et se confondent, les individus et les bâtiments basculent, les variations d'échelles entre les personnages ajoutent à la confusion 
spatiale. Dans cette scène de violence et de torture, les corps sont exagérément mutilés et déformés pour évoquer les conséquences tragiques de la guerre.

Cette violence est toutefois plus caractéristique de certains Muralistes mexicains qui ont marqué Pollock. En 1933, il a vu Diego Rivera peindre sa fresque au Rockefeller Center. A New York, il a également regardé travailler José Clemente Orozco, dont les compositions souvent centrifuges et all-over l'ont impressionné. Le format de Untitled rappelle les tensions et déformations de la fresque d'Orozco réalisée en 1934 au Palacio de Bellas Artes de Mexico ; quant aux fresques d'Orozco à Pomona et ??? Dartmouth College en 1930, leurs formes stylisées et variations d'échelle ont également frappé Pollock. Celui-ci a d'autre part participé à l'Atelier / Laboratoire expérimental du peintre mexicain radical David Alfaro Siqueiros en 1936 et vu son Collective Suicide (1936) au MoMA en 1937.

11 Untitled présente à la fois un tourbillon d'émotions, d'énergies et d'influences ; c'est une œuvre de transition entre l'Europe et les Etats-Unis, entre figuration et abstraction, entre les années trente et les années quarante. Cela explique que les commissaires l'aient choisie; cela n'explique pas qu'elle ait été la seule toile de Pollock dans cette exposition, accrochée en fin de parcours.

\section{Accrochage et affichage}

12 On peut se demander quels autres tableaux de Pollock auraient été à leur place dans The Age of Anxiety. Pollock est presque uniquement connu comme le géant de l'expressionnisme abstrait, pour ses compositions abstraites et ses innovations formelles, mais il n'est pas né Jack the Dripper, et cette exposition aurait pu être l'occasion de montrer le parcours de cet artiste au cours des années trente, avant qu'il ne fasse la couverture des magazines.

13 Il a suivi les cours de Thomas Hart Benton à l'Art Students League et a travaillé à ses côtés dans le cadre du WPA. Un Pollock Régionaliste aurait pu être une révélation pour des visiteurs peu familiers de ses premières œuvres - d'autant plus que cette exposition, rebaptisée America After the Fall'1, a été montrée à la Royal Academy de Londres de février à juin 2017, quelques semaines seulement après une impressionnante exposition sur l'Ecole de New York (septembre 2016-janvier 2017).

On aurait pu mettre en évidence les rapports étroits que Pollock entretenait avec Thomas Hart Benton en plaçant certaines œuvres en regard : Going West (1934-35, National Museum of American Art, Washington, D.C.) aurait pu se trouver dans la salle consacrée au "Retour à la Terre ", à côté d'œuvres dépeignant le Dust Bowl ou les travaux de la ferme. Cette toile de Pollock, dépeint un exode vers l'Ouest américain : ses personnages miséreux, de même que les montagnes et les nuages, sont emportés par un mouvement circulaire; les animaux ploient sous leur charge et négocient un terrain accidenté ; la lumière est blafarde, la palette limitée et sombre, le bleu et le vert sont froids. Ce tableau aurait pu illustrer le propos des commissaires : montrer comment les artistes des années trente ont représenté les conditions de vie difficiles pendant la Grande Dépression.

15 Dans la salle « Puissance industrielle et retour à la terre » on pouvait voir The Cotton Pickers de Thomas Hart Benton (1945, Chicago Art Institute). On aurait pu y adjoindre le tableau de Pollock sur le même sujet, Cotton Pickers (1935, Albright-Knox Museum, 
Buffalo, N.Y.). Cela aurait permis d'établir un contraste entre les deux tableaux, éloignés de dix ans : les travailleurs de Pollock sont anonymes, sans visage, courbés dans un paysage drainé de ses couleurs, sous un ciel tourmenté - une misère universelle entre Millet et Van Gogh. Les ramasseurs de coton de Benton sont miséreux eux aussi et un enfant dort à l'ombre d'une tente de fortune, mais les visages de ces travailleurs africains-américains sont différenciés, leurs traits sont définis, et les personnages sont reliés par le geste d'une femme qui distribue de l'eau, ainsi que par des aplats de rouge qui donnent du relief à la composition.

Autant que les considérations scientifiques, la logistique joue son rôle dans la sélection des œuvres - il aurait probablement été difficile et coûteux d'emprunter plusieurs Pollock, surtout pour une exposition itinérante, car les différents musées hésitent parfois à se défaire de leurs tableaux les plus célèbres pendant plusieurs mois. Untitled appartient à l'Art Institute de Chicago, co-organisateur de l'exposition, et donc bien disposé à prêter une œuvre qui servira le rayonnement de sa collection. La collection de l'Art Institute compte dix autres œuvres de Pollock, toutes datant des années 1940, 1950 ou 1960. Pour exposer un autre Pollock des années trente, il aurait donc fallu l'emprunter à une autre institution.

Quoi qu'il en soit, même si Untitled était le seul tableau de Pollock disponible, il aurait sans doute pu être davantage intégré à l'exposition. Par exemple à côté d'un tableau d'Ilya Bolotowsky, Study for the Hall of Medical Sciences Mural at the 1939 World's Fair in New York (1938-39, The Art Institute of Chicago) ${ }^{2}$, ou à proximité du tableau d'Arthur Dove Swing Music (Louis Armstrong) (1938, The Art Institute of Chicago). Ces œuvres auraient alors pu susciter une discussion autour de la question de l'abstraction aux Etats-Unis, "l'exploration de l'abstraction comme nouveau langage universel» (panneau introductif) ou de l'influence des artistes modernes européens sur la peinture américaine, Miró pour Bolotowsky, Picasso pour Pollock. L'avant-dernière salle de l'exposition, «Cauchemars et réalités » aurait également pu accueillir Untitled, et cette juxtaposition aurait alors mis en avant la dimension psychanalytique de l'œuvre.

Pourtant c'est en fin de parcours que les commissaires ont placé ce tableau; ils en font ainsi le fruit et l'aboutissement d'une décennie, et le début d'une nouvelle ère. Untitled est accroché à côté de la toile de Hopper, Gas (1940) pour annoncer « les deux pôles de la peinture d'après-guerre : l'un ancré dans le réalisme, l'autre dans l'abstraction. » Le texte d'introduction précise aussi que Gas a été choisi parce qu'il « inclut la présence quotidienne de la culture commerciale moderne, tendance reprise vingt ans plus tard par les artistes du Pop Art.» On pourrait néanmoins arguer que l'iconographie commerciale qui apparaît dans la toile de Charles Green Shaw présentée au début de l'exposition, Wrigley's (1937, Art Institute of Chicago), ou dans certaines toiles de Stuart Davis ou de Gerald Murphy, a davantage d'affinités avec le Pop Art - Gas n'est pas vraiment un précurseur de la Standard Station d'Ed Ruscha, et Hopper aurait rechigné à être considéré comme proto-Pop. Comme Hopper, Pollock est ici convoqué pour parfaire un roman national : «L'exploration de l'abstraction comme nouveau langage universel trouve à la fin de la décennie avec les premiers chefs-d'œuvre de Jackson Pollock, un des premiers accomplissements proprement américains. »

L'un des nombreux mérites de cette exposition était de faire découvrir au grand public une période et des artistes relativement inconnus. Inclure des œuvres iconiques comme American Gothic ou des figures célèbres comme Georgia O'Keeffe ou Edward Hopper permettait au visiteur de conserver des repères parmi des noms peu familiers. 
Toutefois, clore l'exposition sur Pollock, lui attribuer une place cruciale après avoir exclu ses œuvres réalistes, c'est paradoxalement omettre de l'inscrire dans une lignée américaine, dans un passé riche et complexe; on le prive ainsi de sa propre histoire. En faisant commencer la carrière de Pollock à la fin des années quarante, on affirme surtout qu'après la Seconde Guerre mondiale New York a bel et bien volé l'idée d'art moderne.

\section{BIBLIOGRAPHIE}

\section{Toiles mentionnées}

Jackson Pollock, Untitled (c.1938-1941) :

https://theredlist.com/media/database/fine_arts/arthistory/painting/peinture_abstraite/ jackson-pollock/033-jackson-pollock-theredlist.jpg

Edward Hopper, Gas (1940):

https://www.moma.org/collection/works/80000

Jackson Pollock, Masqued Image (1938) :

http://www.themodern.org/collection/masqued-image/1211

Pablo Picasso, Guernica (1937) :

http://www.museoreinasofia.es/en/collection/artwork/guernica

Jackson Pollock, The Moon Woman (1942) :

https://www.guggenheim.org/artwork/3473

Thomas Hart Benton, People of Chilmark (Figure Composition) :

https://commons.wikimedia.org/wiki/File:People-of-Chilmark-Benton-1920-lrg.jpg

Thomas Hart Benton, Invasion (1942) : https://i.imgur.com/Z1HjKzm.jpg

José Clemente Orozco, fresque, Palacio de Bellas Artes de Mexico, 1934 :

http://c8.alamy.com/comp/EDNG59/jose-clemente-orozco-fresco-painting-in-the-bellas-artesmuseum-mexico-EDNG59.jpg

José Clemente Orozco, fresques, Pomona, Dartmouth College, 1930 :

https://www.pomona.edu/museum/sites/museum.pomona.edu/files/images/collections/ prometheus-1930-2017-ss-4.jpg

David Alfaro Siqueiros, Collective Suicide (1936) :

https://www.moma.org/collection/works/79146

Jackson Pollock, Going West (1934-35) :

https://i.pinimg.com/originals/fb/4e/13/fb4e13dac44e54cf2d03d5207c6f83ec.jpg

Thomas Hart Benton, The Cotton Pickers (1945) :

http://www.artic.edu/aic/collections/artwork/217201 
Jackson Pollock, Cotton Pickers (1935) :

https://www.albrightknox.org/artworks/1974829-cotton-pickers

Ilya Bolotowsky, Study for the Hall of Medical Sciences Mural at the 1939 World's Fair in New York

(1938-39) :

http://www.artic.edu/aic/collections/artwork/93779?search_no=7\&index=1

Arthur Dove Swing Music (Louis Armstrong) (1938) :

http://www.artic.edu/aic/collections/artwork/65901

Charles Green Shaw, Wrigley's (1937) :

http://www.artic.edu/aic/collections/artwork/53042

Ed Ruscha, Standard Station :

http://blogs.getty.edu/pacificstandardtime/files/2011/07/gm_326202ex1_d.jpg

\section{NOTES}

1. Ce titre était déjà celui de l'exposition à l'Art Institute de Chicago, du 5 juin au 18 septembre 2016.

2. Ce tableau fait l'objet d'une analyse dans ce dossier. Voir Véronique Ha Van, «L'abstraction au cœur de l'Exposition ».

\section{AUTEUR}

\section{CATHERINE MARCANGELI}

Université Paris VII - Denis Diderot 